\title{
Potential kleptoparasitism-scavenging interactions between crabs and shell-boring gastropods feeding on bivalve prey: a global survey
}

\author{
BRADY K. QUINN* \\ Department of Biological Sciences, University of New Brunswick, Saint John, NB Canada E2L 4L5
}

Received 16 November 2019; Accepted 10 February 2020 Responsible Editor: Akira Asakura

doi: $10.3800 /$ pbr. 15.132

\begin{abstract}
Shell-boring gastropod molluscs and crabs are important predators of many bivalve molluscs, and impact bivalve populations and food webs. When gastropods and crabs target the same bivalve prey, complex interactions between them arise that mediate their impacts. Kleptoparasitism and scavenging (K-S) interactions result when one predator, usually the gastropod, loses or abandons its prey, which is then stolen or scavenged by the other. Such interactions have thus far only been reported among nine crab-gastropod species groups when they fed on shared bivalve prey, but potentially occur among many others. This study used the Global Biotic Interactions (GloBI) database to search for records of shell-boring gastropods and crabs preying on the same bivalve to identify tritrophic bivalve-crab-gastropod systems in which K-S interactions may occur. In total, 871 such systems were found, which included 58 bivalve species that were each eaten by 1-8 species of shell-boring gastropods and 1-19 species of crabs. In the vast majority of these systems (>99\%), no previous report of K-S interactions has been published, although in 334 cases the gastropod and crab have been reported to undergo competitive or predator-prey interactions, which may indicate that K-S and other complex interactions occur among them. The tritrophic systems identified in the present survey should be investigated in future studies to determine whether the identified crab and gastropod taxa do in fact undergo K-S interactions. Doing so will enable the development of a theoretical framework for assessing the occurrence, evolution, utilization, and impacts of these interactions in natural systems.
\end{abstract}

Key words: Bivalve, crab, shell-boring gastropod, kleptoparasitism, scavenging

\section{Introduction}

Bivalve molluscs (clams, cockles, mussels, oysters, scallops, etc.) are filter-feeding organisms that form the bases of many aquatic food webs in both marine and freshwater environments (Seed 1976, Cockrell et al. 2015). Bivalves are important food sources for a wide variety of secondary and higher-level consumers, including decapod crustaceans, gastropod molluscs, polychaete worms, fishes, birds, humans, and many others (Seed 1976, Hamilton 2000, Morissette \& Himmelman 2000, Trussell et al. 2017), and also play roles in controlling habitat structure (Enderlein \& Wahl 2004) and community diversity (Boudreau \& Hamilton 2012). Although predation on bivalves by vertebrates can strongly influence their population dynamics (Hamilton 2000, Daleo et al. 2005, Cockrell et al.

*Corresponding author: Brady K. Quinn; E-mail, bk.quinn@unb.ca
2015), invertebrate consumers also play comparably important roles as bivalve predators (Vadas et al. 1994, Trussell et al. 2003, Aschaffenberg 2008, Wong et al. 2012, Morgan et al. 2016, Boudreau \& Hamilton 2012, Bolton et al. 2019). However, the specific trophic roles and impacts of invertebrate predators on their bivalve prey depend on the interactions that occur among them. This is particularly apparent in the case of intraguild predation (IGP), when one predator (called the 'intermediate predator') is also a potential prey item of another (the 'top predator') that feeds on the same prey species (Polis \& Holt 1992, Arim \& Marquet 2004). IGP frequently occurs among predators of bivalves, most notably between crabs (Crustacea: Decapoda: Brachyura or Anomura) and shell-drilling or -boring gastropod molluscs, such as whelks, wherein these gastropod predators may also be preyed on by crab predators (Vadas et al. 1994, Aschaffenberg 2008, Boudreau et al. 2013).

IGP leads to complex and non-additive interspecific 
competitive interactions (Sih et al. 1985, Quinn et al. 2012), and in many cases has also led to the evolution of particular responses by the intermediate predator to avoid being eaten by the top predator that affect its feeding on their shared prey (Trussell et al. 2003, Aschaffenberg 2008). Such responses are known as trait-mediated indirect interactions (TMIIs), because some trait(s) of the intermediate predator (e.g., shell thickness, shell shape, feeding rate, etc.) is altered without any direct (e.g., predator-prey) interaction occurring between the two predatory species (Trussell et al. 2003, 2017, Morgan et al. 2016); when the intermediate predator's behaviour is changed, this TMII is termed a behaviourally mediated indirect interaction (BMII) (Dill et al. 2003). A typical example of a BMII is when gastropods feed less often and more slowly when they detect the presence (e.g., smell) of crabs in their environment (Vadas et al. 1994, Powers \& Kittinger 2002, Trussell et al. 2003, Boudreau et al. 2018). This reduced feeding rate results in greater impairment of gastropod feeding than would result from competition with crabs alone (Quinn et al. 2012, Wong et al. 2012), leading to less gastropod-induced mortality of bivalve prey (Hamilton 2000, Boudreau \& Hamilton 2012) and other effects (Quinn et al. 2012, Wong et al. 2012, Morgan et al. 2016). IGP scenarios also create the possibility of there being further complex interactions between predators of bivalves, including positive interactions or facilitation [when one predator feeds more in response to the other's presence (Daleo et al. 2005, Wong et al. 2012)], as well as kleptoparasitism and scavenging interactions (Boudreau et al. 2013, Quinn \& Boudreau 2016).

Kleptoparasitism refers to an interaction in which one organism (the kleptoparasite) obtains some resource already acquired by another (the host) by stealing it (Barnard \& Sibly 1981), including when one predator steals a prey item acquired by another (Iyengar 2008). On the other hand, scavenging occurs when an organism feeds on dead animal biomass in its environment (Barnard \& Sibly 1981), and can include cases wherein one predator kills and begins to eat a prey item, but then for some reason (e.g., satiation) abandons it, and it is then eaten by another predator (Morton 2011, Quinn \& Boudreau 2016). Both are opportunistic trophic strategies that allow a consumer to obtain food at less cost (search and handling time, risk, etc.) than if they preyed on it themselves (Barnard \& Sibly 1981, Iyengar 2008, Quinn et al. 2012; Boudreau et al. 2013). In the case of kleptoparasitism, the host consumer also suffers a heavy loss due to the theft of its food, representing an extreme case of interference competition (Smallegange et al. 2006). Conversely, one predator scavenging on another's abandoned prey does not inflict any cost on the initial predator (Quinn \& Boudreau 2016 and references therein).

In a recent series of studies, such interactions were observed between green crabs [Carcinus maenas (L., 1758)] and dogwhelks ([Nucella lapillus (L., 1758)] feeding on blue mussels (Mytilus spp.), both in the crab's native (Mor- ton 2011) and invasive ranges (Quinn et al. 2012, Boudreau \& Hamilton 2012, Boudreau et al. 2013, Quinn \& Boudreau 2016, Boudreau et al. 2018), which had interesting implications to the species involved. These mussels were initially attacked by one or more dogwhelks, which weakened them and caused their shells to gape; this, in turn, made it easier for a crab to break into the weakened mussel and consume it (Quinn et al. 2012). Mussel weakening by dogwhelk attacks also allowed relatively small crabs to feed on relatively larger mussels than they could have broken open under their own power (Enderlein et al. 2003, Boudreau et al. 2013). For example, Quinn et al. (2012) reported that crabs used kleptoparasitism to eat mussels that were ca. $2.5 \mathrm{~mm}$ larger in shell length than they could consume alone, representing an increase in potential mussel biomass consumption of $18 \%$ (Quinn et al. 2012). When a crab picked up a mussel while it was still being consumed by a dogwhelk, dislodged the dogwhelk, and then proceeded to eat the mussel, this represented kleptoparasitism, and represented a substantial gain to the crab and loss to the dogwhelk (Quinn \& Boudreau 2016); when a whelk became sated while eating a mussel and abandoned it, and that mussel was then found and eaten by a crab, this represented scavenging, and was solely a gain to the crab with no loss to the sated dogwhelk (Morton 2011, Quinn \& Boudreau 2016). Kleptoparasitism increased crab predation rates and decreased dogwhelk predation rates by 22.8 $28.6 \%$ and $65.4-67.3 \%$, respectively, and resulted in dogwhelks being $43.3-45.0 \%$ less satiated after 2 weeks, while scavenging increased crab feeding by a further $3.5-3.9 \%$ without significantly impacting whelk feeding or satiation (Quinn \& Boudreau 2016). Both of these interactions between gastropods and crabs on the same bivalve prey thus have potentially strong impacts on predator feeding rates. However, they can only be distinguished from one another if they are directly observed, whereas after the fact the indirect signs that they occurred (a dead/empty mussel shell with signs of both gastropod drilling/boring and crab chipping/crushing) are the same (Boudreau et al. 2013, Quinn \& Boudreau 2016). In many studies, though, only the latter of these types of observations is possible. Given the similarity of these unique tritrophic (bivalve-crab-gastropod) interactions to one another, they are thus referred to hereinafter collectively as kleptoparasitism-scavenging (K-S) interactions.

These K-S interactions between green crabs and dogwhelks feeding on the same blue mussel prey represented a component of the IGP, TMII/BMII, and trophic interactions in this system, with probable impacts on mussel survival and population growth, and subsequently on mussel bed ecosystem dynamics (Boudreau \& Hamilton 2012, Cockrell et al. 2015, Trussell et al. 2017), and yet they had not been reported previously. However, this tritrophic system is by no means unique, as many bivalves throughout the world are consumed by both shell-drilling gastropods and crabs (Carriker \& Williams 1978, Kowalewski 2002, 
Ishida 2004, Klompmaker et al. 2019). A handful of previous studies on other bivalve-crab-gastropod systems have reported the occurrence of such K-S interactions within these species' native ranges (Table 1 and references therein), but overall these types of interactions do not appear to have been reported much. Given that many crabs are intelligent and aggressive predators capable of learning and adopting new opportunistic foraging strategies (Enderlein et al. 2003, Smallegange et al. 2006, Quinn et al. 2012), it is likely that this represents the results of the underreporting or missed observations of the occurrence of these interactions, rather than their absence.

Given the above, the objective of the present study was to conduct a global survey of the trophic overlap between crab and gastropod predators feeding on bivalve prey to identify pairs or groups of crab and gastropod species that may undergo K-S interactions. The information collected supplements the relatively sparse data in the literature on crab-gastropod K-S interactions, and provides material for potential future reviews of crab-gastropod trophic overlap (e.g., concerning IGP and BMIIs/TMIIs). Further, the survey results identify crab and gastropod predators that should be the foci of future studies to confirm whether they do indeed undergo K-S interactions, and if so what their consequences are.

\section{Materials and Methods}

The Global Biotic Interactions (GloBI) database (Poelen et al. 2014; accessed online at: https://www.globalbioticin teractions.org) is an open access and open source online tool that collects published and user-submitted reports of species interactions. This database can be queried to determine with which other organisms a particular taxon (e.g., species) interacts as a predator, prey, parasite, host, and so on. This database is thus a useful tool for scientific studies and reviews of species interactions, and was used in the present study. The GloBI database was queried on 24 May 2019 to obtain lists of the bivalve mollusc (Mollusca: Bivalvia) species consumed by different groups of crab and shell-boring gastropod species. For crabs, both the decapod crustacean infraorders Anomura (hermit crabs and their relatives) and Brachyura (true crabs) were considered. For gastropods, those extant gastropod families or groups considered important shell-boring or -drilling groups (sen$s u$ Kowalewski 2002 and references therein) and/or commonly termed 'whelks' were considered, which included the following groups: the families Buccinidae, Capulidae, Cassidae, Eulimidae, Marginellidae, Muricidae, Nassariidae, Naticidae, Ranellidae, and Trochidae, as well as the order Nudibranchia and the informal group 'Pulmonata.'

The 'Browse' tool on the GloBI website was used to que-

Table 1. Previous reports of K-S interactions between crabs and shell-boring gastropods feeding on shared bivalve prey. The cited studies were found from BIOSIS Citation Index/Web of Science (Clarivate Analytics, 2019) searches performed on 23 May 2019 for ['kleptoparasit*' or 'scaveng*'] and ['crab' or 'crustacea*'] and ['whelk' or 'snail' or 'gastropod*']. Entries are listed alphabetically by prey species name, then by kleptoparasite/scavenger species name and host/primary predator species names. All species were studied in their native range unless indicated otherwise [in square brackets following their name].

\begin{tabular}{|c|c|c|c|c|}
\hline Prey $(\mathrm{K} / \mathrm{S})$ & Kleptoparasite/scavenger & Host/primary predator & Location & Source(s) \\
\hline $\begin{array}{l}\text { Crassostrea virginica } \\
\text { (Gmelin, 1791) } \\
\text { (S, K mentioned) }\end{array}$ & $\begin{array}{l}\text { Stramonita haemastoma } \\
\quad(\mathrm{L} ., 1767)\end{array}$ & $\begin{array}{l}\text { Menippe adina Williams \& } \\
\text { Felder, } 1986\end{array}$ & $\begin{array}{l}\text { Dauphin Island, } \\
\text { Alabama, USA }\end{array}$ & $\begin{array}{l}\text { Fodrie et al. } \\
\qquad(2012)\end{array}$ \\
\hline $\begin{array}{l}\text { Hyotissa } \text { cf. fisheri } \\
\text { (Dall, 1914) } \\
\text { (K possible?) }\end{array}$ & $\begin{array}{l}\text { Whelks and crabs } \\
\text { (unspecified) }\end{array}$ & $\begin{array}{l}\text { Vitularia salebrosa } \\
\quad(\text { P. P. King, 1832) }\end{array}$ & Pacific Panama & $\begin{array}{l}\text { Herbert et al. } \\
\qquad(2009)\end{array}$ \\
\hline $\begin{array}{l}\text { Mactromeris polynyma } \\
\text { (Stimpson, } 1860)^{*} \text { and } \\
\text { Mya truncata L., } 1758^{*}(\mathrm{~K})\end{array}$ & $\begin{array}{l}\text { Buccinum undatum } \\
\quad \text { L., } 1758^{*}\end{array}$ & $\begin{array}{l}\text { Hyas araneus (L., 1758)* } \\
\text { and Cancer irroratus } \\
\text { Say, } 1817^{*}\end{array}$ & $\begin{array}{l}\text { Northern Gulf of St. } \\
\text { Lawrence, Quebec, } \\
\text { Canada }\end{array}$ & $\begin{array}{l}\text { Morissette \& } \\
\text { Himmelman } \\
\text { (2000) }\end{array}$ \\
\hline Mytilus edulis L., 1758* (K?) & $\begin{array}{l}\text { Buccinum undatum } \\
\text { L., } 1758^{*}\end{array}$ & $\begin{array}{l}\text { Carcinus maenas } \\
\quad(\mathrm{L} ., 1758)^{*}[\text { Invasive }]\end{array}$ & $\begin{array}{l}\text { New Brunswick, } \\
\text { Canada }\end{array}$ & Walsh (2016) \\
\hline $\begin{array}{l}\text { Mytilus edulis L., 1758* } \\
\text { (K and/or S?) }\end{array}$ & $\begin{array}{l}\text { Carcinus maenas } \\
(\mathrm{L} ., 1758)^{*} \\
\text { [Invasive] }\end{array}$ & Nucella lapillus (L., 1758)* & $\begin{array}{l}\text { New Brunswick, } \\
\text { Canada }\end{array}$ & $\begin{array}{l}\text { Quinn et al. (2012), } \\
\text { Boudreau et al. } \\
\text { (2013) }\end{array}$ \\
\hline $\begin{array}{l}\text { Mytilus galloprovincialis } \\
\text { Lamarck, 1819*(S) }\end{array}$ & $\begin{array}{l}\text { Carcinus maenas } \\
\quad(\mathrm{L} ., 1758)^{*}\end{array}$ & Nucella lapillus (L., 1758)* & United Kingdom & Morton (2011) \\
\hline $\begin{array}{l}\text { Ruditapes philippinarum } \\
\qquad(\text { Adams \& Reeve, } 1850)^{*}(\mathrm{~K})\end{array}$ & $\begin{array}{l}\text { Portunus trituberculatus } \\
\quad \text { (Miers, 1876)* }^{*}\end{array}$ & $\begin{array}{l}\text { Rapana venosa } \\
\quad(\text { Valenciennes, 1846)* }\end{array}$ & Qingdao, China & $\begin{array}{l}\text { Liu et al. } \\
\quad(2017,2019)\end{array}$ \\
\hline $\begin{array}{l}\text { Ruditapes philippinarum } \\
\text { (Adams \& Reeve, 1850) } \\
\text { carrion (K?) }\end{array}$ & $\begin{array}{l}\text { Reticunassa festiva } \\
\quad \text { (Powys, 1835) }\end{array}$ & $\begin{array}{l}\text { Diogenes edwardsii } \\
\quad \text { (De Haan, 1849) }\end{array}$ & Hong Kong & $\begin{array}{l}\text { Morton \& Yuen } \\
\quad(2000)\end{array}$ \\
\hline
\end{tabular}

Note: an '*' indicates groups of interacting species that were recovered in the 'clusters' found in the main survey (see Table 3 in Results). 
ry what kind of 'Bivalvia' each crab or gastropod group ('Anomura,' 'Brachyura, 'Buccinidae,' etc.) 'eats,' the results of which included which predator taxon 'eats,' 'preys on,' and is a 'parasite of' which bivalve taxa. The results of these queries were downloaded as .csv files, and then compiled into combined crab and gastropod datasets to identify crabs and gastropods that eat the same bivalve prey. Within these query results, records of members of 4 gastropod families (Buccinidae, Muricidae, Naticidae, and Ranellidae) consuming the same bivalve prey species as one or more crabs were found. However, no crab-gastropod trophic overlap involving members of the other 8 queried gastropod groups was found, and thus these groups could not be considered further herein. It should also be noted that the only gastropod species that were found in searches for 'Ranellidae' to eat the same bivalves as crabs are actually former members of this family that are currently classified within a different family, Cymatiidae, and one of the species of 'Buccinidae' found has been reclassified as a member of the family Pisaniidae; however, since these species are still shell-boring gastropods, the results for them were retained.
Although records for some higher taxonomic levels were initially obtained in query results (e.g., members of a class, order, superfamily, or family were listed as eating/being eaten by another taxon, without specifying the specific genus or species involved), these were not retained as they were deemed too vague. Therefore, only records at the species or genus level were retained and considered further. To avoid redundancy and because the names of some taxa listed in the GloBI database were out of date, the World Register of Marine Species (WoRMS: http://www.marine species.org/; WoRMS Editorial Board 2019) was consulted to determine the proper classification and most up-to-date name of each species or genus as of 28 May 2019. The combined crab and whelk datasets were next compared to identify the bivalve species that were shared between them (i.e., cases of trophic overlap, where the same bivalve was eaten by at least one crab and at least one whelk). All bivalves not shared between these two datasets were then discarded and not considered further. Thus, 58 bivalve species were retained that were shared between the crab and gastropod datasets, and each of these was defined as the

Table 2. Species of crabs that were frequently listed as feeding on the same bivalve prey in the GloBI database survey, defined herein as 'Crab Groups.'

\begin{tabular}{|c|c|c|c|c|c|}
\hline \multicolumn{2}{|r|}{ Species } & \multicolumn{4}{|c|}{ Crab Group } \\
\hline & & $\mathrm{I}(\mathrm{n}=11)$ & II $(n=15)$ & III $(n=19)$ & IV $(n=9)$ \\
\hline An & Pagurus brevidactylus (Stimpson, 1859) & V & & V & \\
\hline An & Pagurus stimpsoni (A. Milne-Edwards \& Bouvier, 1893) & V & & V & \\
\hline $\mathrm{Br}$ & Acanthocyclus albatrossis Rathbun, 1898 & V & & V & V \\
\hline $\mathrm{Br}$ & Calappa ocellata Holthuis, $1958^{\mathrm{ABCDEF}}$ & V & & V & V \\
\hline $\mathrm{Br}$ & Cyclozodion angustum (A. Milne-Edwards, 1880) ${ }^{\mathrm{ABCDEF}}$ & V & & V & V \\
\hline $\mathrm{Br}$ & Panopeus occidentalis Saussure, $1857^{\mathrm{ABCDEF}}$ & V & & V & V \\
\hline $\mathrm{Br}$ & Pilumnus dasypodus Kingsley, 1879 $\mathrm{ABCDEF}$ & V & & V & V \\
\hline An & Paguristes grayi Benedict, 1901 & V & V & V & V \\
\hline $\mathrm{Br}$ & Pilumnus lacteus Stimpson, $1871^{\mathrm{ABCDEF}}$ & V & V & V & V \\
\hline $\mathrm{Br}$ & Pilumnus marshi Rathbun, $1901^{\mathrm{ABCDEF}}$ & V & V & V & V \\
\hline $\mathrm{Br}$ & Pilumnus pannosus Rathbun, $1896^{\mathrm{ABCDEF}}$ & V & V & V & V \\
\hline $\mathrm{Br}$ & Garthiope barbadensis (Rathbun, 1921) ${ }^{\mathrm{BC}}$ & & V & V & \\
\hline $\mathrm{Br}$ & Pilumnus diomedeae Rathbun, $1894^{\mathrm{BC}}$ & & V & V & \\
\hline $\mathrm{Br}$ & Pilumnus gemmatus Stimpson, $1860^{\mathrm{BC}}$ & & V & V & \\
\hline $\mathrm{Br}$ & Pilumnus holosericus Rathbun, $1898^{\mathrm{BC}}$ & & V & V & \\
\hline $\mathrm{Br}$ & Pilumnus longleyi Rathbun, $1930^{\mathrm{BC}}$ & & V & V & \\
\hline $\mathrm{Br}$ & Pilumnus reticulatus Stimpson, $1860^{\mathrm{BC}}$ & & V & $\checkmark$ & \\
\hline $\mathrm{Br}$ & Pilumnus sayi Rathbun, $1897^{\mathrm{BC}}$ & & V & V & \\
\hline $\mathrm{Br}$ & Scopolius nuttingi (Rathbun, 1898) & & V & V & \\
\hline $\mathrm{Br}$ & Carpilius corallinus (Herbst, 1783) & & V & & \\
\hline An & Paguristes cadenati Forest, 1954 & & V & & \\
\hline An & Paguristes tortugae Schmitt, 1933 & & V & & \\
\hline
\end{tabular}

Notes: Species labels in the left-hand column correspond to the infraorders Anomura ('An') and Brachyura ('Br'). Superscript letters indicate

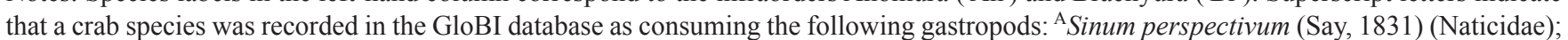
${ }^{\mathrm{B}}$ Monoplex pilearis (L., 1758) (Cymatiidae, formerly Ranellidae); ${ }^{\mathrm{C}}$ Monoplex vespaceus (Lamarck, 1822) (Cymatiidae, formerly Ranellidae); ${ }^{\mathrm{D}}$ Dermomurex pauperculus (C. B. Adams, 1850) (Muricidae); ${ }^{\mathrm{E}}$ Cymatium femorale (L., 1758) (Cymatiidae, formerly Ranellidae); ${ }^{\mathrm{F}}$ Monoplex nicobaricus (Röding, 1798) (Cymatiidae, formerly Ranellidae). 
Table 3. Crabs and shell-boring gastropods potentially undergoing K-S interactions when feeding on shared bivalve prey according to a survey of the GloBI database. Trophic 'clusters' (numbered in the rightmost column) are listed alphabetically by bivalve species name, then by crab species name and gastropod species name.

\begin{tabular}{|c|c|c|c|}
\hline Crab consumer & Bivalve consumed & Gastropod consumer & $\#$ \\
\hline Crab Group I $(\mathrm{n}=11)^{\mathrm{A}}$ & Americardia media (L., 1758) & Sinum perspectivum $(\text { Say, } 1831)^{\mathrm{A}}$ & 1 \\
\hline Crab Group I $(\mathrm{n}=11)^{\mathrm{A}}$ & Anadara notabilis (Röding, 1798) & Sinum perspectivum $(\text { Say, } 1831)^{\mathrm{A}}$ & 2 \\
\hline Crab Group I $(n=11)^{A}$ & Anodontia alba Link, 1807 & Sinum perspectivum $(\text { Say, } 1831)^{\mathrm{A}}$ & 3 \\
\hline Crab Group II $(\mathrm{n}=15)^{\mathrm{AB}}$ & Arca zebra Swainson, 1833 & $\begin{array}{l}\text { Monoplex pilearis }(\mathrm{L} ., 1758)^{\mathrm{A}} \\
\text { Monoplex vespaceus }(\text { Lamarck, 1822) })^{\mathrm{B}}\end{array}$ & 4 \\
\hline Crab Group II $(\mathrm{n}=15)^{\mathrm{AB}}$ & Arcopsis adamsi (Dall, 1886) & $\begin{array}{l}\text { Monoplex pilearis }(\mathrm{L} ., 1758)^{\mathrm{A}} \\
\text { Monoplex vespaceus }(\mathrm{Lamarck}, 1822)^{\mathrm{B}}\end{array}$ & 5 \\
\hline $\begin{array}{l}\text { Halicarcinus whitei (Miers, 1876) } \\
\text { Hemigrapsus crenulatus }(\mathrm{H} . \text { Milne } \\
\quad \text { Edwards, 1837) } \\
\text { Hemigrapsus } \text { sp. } \\
\text { Hem } \\
\text { Hemiplax hirtipes Heller, } 1865^{\mathrm{ABC}} \\
\text { Pinnotheres sp. }\end{array}$ & Austrovenus stutchburyi (W. Wood, 1828) & $\begin{array}{l}\text { Buccinulum pertinax (Martens, 1878) } \\
\text { Cominella glandiformis (Reeve, 1847) }\end{array}$ & 6 \\
\hline Macrophthalmus sp. ${ }^{\mathrm{A}}$ & Austrovenus sp. & Cominella sp. ${ }^{\mathrm{A}}$ & 7 \\
\hline Crab Group II $(\mathrm{n}=15)^{\mathrm{AB}}$ & Barbatia candida (Helbling, 1779) & $\begin{array}{l}\text { Monoplex pilearis }(\mathrm{L} ., 1758)^{\mathrm{A}} \\
\text { Monoplex vespaceus }(\text { Lamarck, } 1822)^{\mathrm{B}}\end{array}$ & 8 \\
\hline Crab Group II $(\mathrm{n}=15)^{\mathrm{AB}}$ & Barbatia domingensis (Lamarck, 1819) & $\begin{array}{l}\text { Monoplex pilearis }(\mathrm{L} ., 1758)^{\mathrm{A}} \\
\text { Monoplex vespaceus }(\text { Lamarck, 1822) }\end{array}$ & 9 \\
\hline $\begin{array}{l}\text { Carcinus maenas }(\mathrm{L} ., 1758)^{\mathrm{AB}} \\
\text { Pagurus bernhardus }(\mathrm{L} ., 1758)\end{array}$ & Cerastoderma edule (L., 1758) & $\begin{array}{l}\text { Hexaplex trunculus }(\mathrm{L} ., 1758)^{\mathrm{A}} \\
\text { Nucella lapillus }(\mathrm{L} ., 1758)^{\mathrm{B}}\end{array}$ & 10 \\
\hline Crab Group II $(\mathrm{n}=15)^{\mathrm{AB}}$ & Chama macerophylla Gmelin, 1791 & $\begin{array}{l}\text { Monoplex pilearis }(\mathrm{L} ., 1758)^{\mathrm{A}} \\
\text { Monoplex vespaceus }(\text { Lamarck, 1822) }\end{array}$ & 11 \\
\hline Crab Group II $(\mathrm{n}=15)^{\mathrm{AB}}$ & Chama sarda Reeve, 1847 & $\begin{array}{l}\text { Monoplex pilearis }(\mathrm{L} ., 1758)^{\mathrm{A}} \\
\text { Monoplex vespaceus }(\text { Lamarck, 1822) }\end{array}$ & 12 \\
\hline Crab Group I $(n=11)^{\mathrm{A}}$ & Chione cancellata (L., 1767) & Sinum perspectivum $(\text { Say, } 1831)^{\mathrm{A}}$ & 13 \\
\hline Crab Group IV $(n=9)^{\mathrm{A}}$ & Clathrolucina costata (d’Orbigny, 1845) & Sinum perspectivum $(\text { Say, } 1831)^{\mathrm{A}}$ & 14 \\
\hline $\begin{array}{l}\text { Cancer sp. } \\
\text { Paralithodes sp. }\end{array}$ & Clinocardium nuttallii (Conrad, 1837) & $\begin{array}{l}\text { Buccinum sp. }{ }^{\mathrm{AB}} \\
\text { Fusitriton oregonensis (Redfield, 1846) }\end{array}$ & 15 \\
\hline Crab Group IV $(n=9)^{\mathrm{A}}$ & Codakia orbicularis (L., 1758) & Sinum perspectivum $(\text { Say, } 1831)^{\mathrm{A}}$ & 16 \\
\hline Callinectes sapidus Rathbun, $1896^{\mathrm{A}}$ & Crassostrea virginica (Gmelin, 1791) & $\begin{array}{l}\text { Busycon carica }(\text { Gmelin, 1791) } \\
\text { Rapana venosa }(\text { Valenciennes, } 1846)^{\mathrm{A}}\end{array}$ & 17 \\
\hline Crab Group IV $(n=9)^{\mathrm{A}}$ & Ctena orbiculata (Montagu, 1808) & Sinum perspectivum $($ Say, 1831) & 18 \\
\hline Crab Group II $(\mathrm{n}=15)^{\mathrm{AB}}$ & Ctenoides scaber (Born, 1778) & $\begin{array}{l}\text { Monoplex pilearis }(\mathrm{L} ., 1758)^{\mathrm{A}} \\
\text { Monoplex vespaceus }(\text { Lamarck, 1822) }\end{array}$ & 19 \\
\hline Crab Group I $(\mathrm{n}=11)^{\mathrm{A}}$ & Dallocardia muricata (L., 1758) & Sinum perspectivum $(\text { Say, } 1831)^{\mathrm{A}}$ & 20 \\
\hline Crab Group I $(n=11)^{\mathrm{A}}$ & $\begin{array}{l}\text { Diplodonta nucleiformis (W. Wagner, } \\
1840 \text { ) }\end{array}$ & Sinum perspectivum $(\text { Say, } 1831)^{\mathrm{A}}$ & 21 \\
\hline Ocypode quadrata (Fabricius, 1787) & Donax variabilis Say, 1822 & Neverita duplicata (Say, 1822) & 22 \\
\hline $\begin{array}{l}\text { Carcinus maenas (L., 1758) } \\
\text { Ovalipes sp. }\end{array}$ & Ensis leei M. Huber, 2015 & $\begin{array}{l}\text { Busycon carica (Gmelin, 1791) } \\
\text { Busycotypus canaliculatus (L., 1758) }\end{array}$ & 23 \\
\hline
\end{tabular}


Table 3. Continued

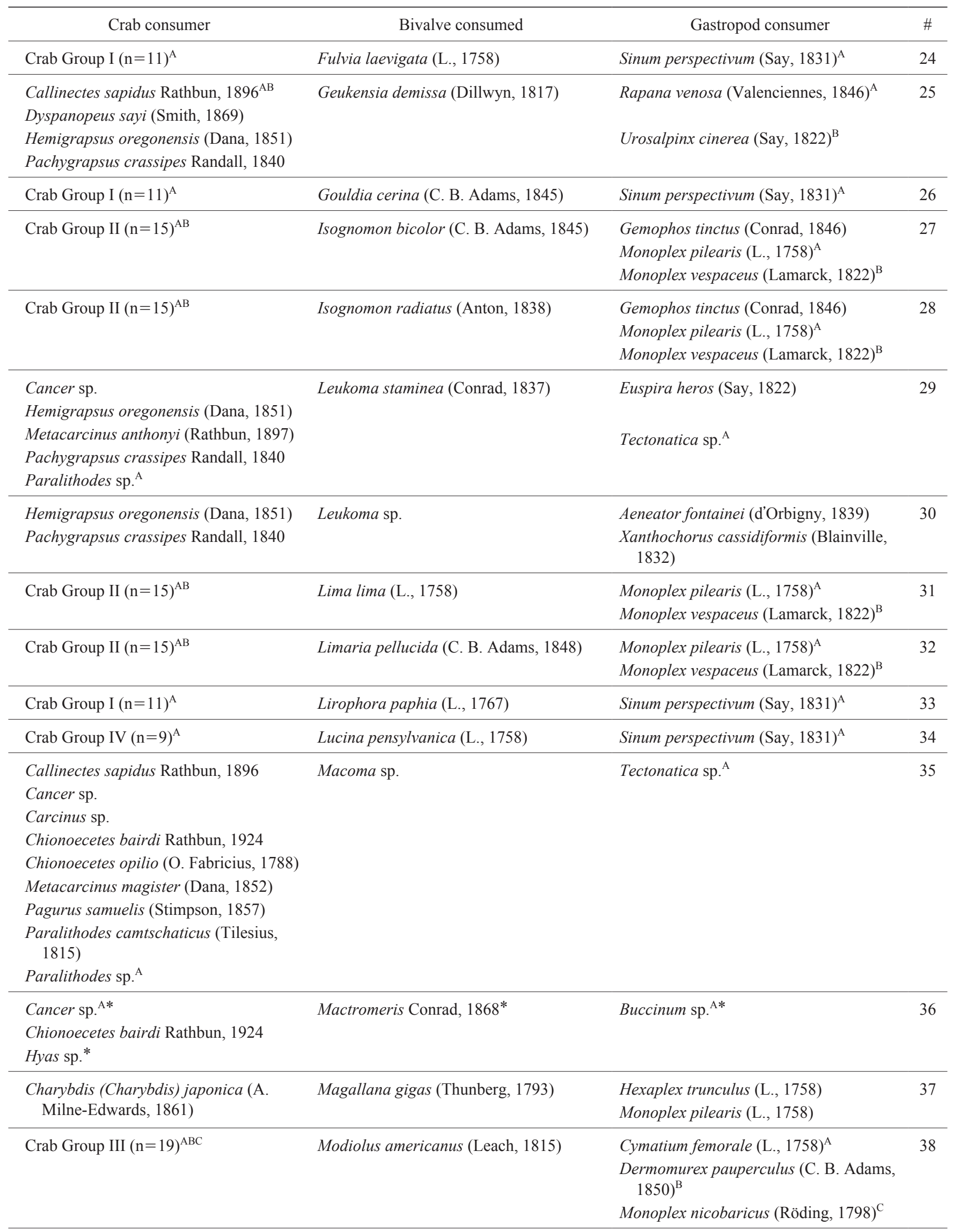


Table 3. Continued

\begin{tabular}{|c|c|c|c|}
\hline Crab consumer & Bivalve consumed & Gastropod consumer & $\#$ \\
\hline $\begin{array}{l}\text { Cancer sp. } \\
\text { Carcinus sp. } \\
\text { Hyas sp. } \\
\text { Pagurus sp. } \\
\text { Paralithodes sp. }\end{array}$ & Mya sp. & Tectonatica sp. ${ }^{\text {В }}$ & 39 \\
\hline Crab Group I $(\mathrm{n}=11)^{\mathrm{A}}$ & Mysia pellucida (Tate, 1891) & Sinum perspectivum $(\mathrm{Say}, 1831)^{\mathrm{A}}$ & 40 \\
\hline $\begin{array}{l}\text { Cancer sp. } \\
\text { Loxorhynchus grandis Stimpson, } 1857\end{array}$ & Mytilus californianus Conrad, 1837 & $\begin{array}{l}\text { Kelletia kelletii (Forbes, 1852) } \\
\text { Nucella emarginata (Deshayes, 1839) } \\
\text { Thais } \text { sp. }\end{array}$ & 41 \\
\hline $\begin{array}{l}\text { Carcinus maenas (L., 1758) } \\
\text { Maja brachydactyla Balss, } 1922 \\
\text { Pagurus bernhardus (L., 1758) } \\
\text { Rhithropanopeus harrisii (Gould, 1841) }\end{array}$ & Mytilus edulis L., 1758* & $\begin{array}{l}\text { Hexaplex trunculus (L., 1758) } \\
\text { Nucella lapillus (L., 1758) } \\
\text { Stramonita canaliculata (Gray, 1839) } \\
\text { Thais sp. }\end{array}$ & 42 \\
\hline $\begin{array}{l}\text { Carcinus aestuarii Nardo, } 1847 \\
\text { Carcinus maenas }(\mathrm{L} ., 1758)^{\mathrm{A} *} \\
\text { Hemigrapsus oregonensis (Dana, 1851) } \\
\text { Liocarcinus depurator (L., 1758) } \\
\text { Necora puber (L., 1767) } \\
\text { Pachygrapsus crassipes Randall, } 1840 \\
\text { Pachygrapsus marmoratus (Fabricius, } \\
\quad 1787)^{\mathrm{B}}\end{array}$ & Mytilus galloprovincialis Lamarck, 1819* & Rapana venosa (Valenciennes, 1846) & 43 \\
\hline $\begin{array}{l}\text { Callinectes sapidus Rathbun, } 1896 \\
\text { Cancer sp. }{ }^{\mathrm{ABC}} \\
\text { Carcinus sp. }^{\mathrm{D} *} \\
\text { Maja squinado (Herbst, 1788) } \\
\text { Pagurus sp. } \\
\text { Paralithodes sp. } \\
\text { Pinnotheres pisum (L., 1767) } \\
\text { Portunus trituberculatus (Miers, 1876) } \\
\text { Pugettia venetiae Rathbun, 1924 }\end{array}$ & Mytilus sp. ${ }^{*}$ & $\begin{array}{l}\text { Buccinum sp. }{ }^{\mathrm{A} *} \\
\text { Fusitriton oregonensis (Redfield, 1846) } \\
\text { Nucella canaliculata (Duclos, 1832) } \\
\text { Nucella emarginata (Deshayes, 1839) } \\
\text { Nucella lamellosa (Gmelin, 1791) } \\
\text { Nucella lima (Gmelin, 1791) } \\
\text { Polinices sp. } \\
\text { Thais sp. }\end{array}$ & 44 \\
\hline $\begin{array}{l}\text { Chionoecetes bairdi Rathbun, } 1924 \\
\text { Chionoecetes opilio (O. Fabricius, 1788) } \\
\text { Paralithodes sp. }\end{array}$ & Nuculana sp. & Tectonatica sp. ${ }^{\mathrm{A}}$ & 45 \\
\hline $\begin{array}{l}\text { Cancer pagurus L., } 1758 \\
\text { Pachygrapsus marmoratus (Fabricius, } \\
\text { 1787) }\end{array}$ & Ostrea edulis L., 1758 & Hexaplex trunculus (L., 1758) & 46 \\
\hline Crab Group I $(n=11)^{A}$ & Papyridea soleniformis (Bruguière, 1789) & Sinum perspectivum $(\text { Say, } 1831)^{\mathrm{A}}$ & 47 \\
\hline Crab Group I $(n=11)^{\mathrm{A}}$ & Periglypta listeri (J.E. Gray, 1838) & Sinum perspectivum $(\text { Say, } 1831)^{\mathrm{A}}$ & 48 \\
\hline Crab Group I $(\mathrm{n}=11)^{\mathrm{A}}$ & Phlyctiderma caelatum (Reeve, 1850) & Sinum perspectivum $(\text { Say, } 1831)^{\mathrm{A}}$ & 49 \\
\hline Crab Group I $(n=11)^{\mathrm{A}}$ & Pitar fulminatus (Menke, 1828) & Sinum perspectivum $(\text { Say, } 1831)^{\mathrm{A}}$ & 50 \\
\hline $\begin{array}{l}\text { Cancer productus Randall, } 1840 \\
\text { Carcinus maenas (L., 1758) } \\
\text { Metacarcinus gracilis (Dana, 1852) } \\
\text { Portunus trituberculatus (Miers, 1876)* }\end{array}$ & $\begin{array}{l}\text { Ruditapes philippinarum (Adams \& } \\
\text { Reeve, } 1850)^{*}\end{array}$ & $\begin{array}{l}\text { Mammilla melanostoma (Gmelin, 1791) } \\
\text { Rapana venosa (Valenciennes, 1846)* }\end{array}$ & 51 \\
\hline Cancer sp. & Saxidomus sp. & Tectonatica sp. & 52 \\
\hline
\end{tabular}


Table 3. Continued

\begin{tabular}{|c|c|c|c|}
\hline Crab consumer & Bivalve consumed & Gastropod consumer & \# \\
\hline Crab Group I $(\mathrm{n}=11)^{\mathrm{A}}$ & Tellina radiata L., 1758 & Sinum perspectivum $($ Say, 1831) & 53 \\
\hline $\begin{array}{l}\text { Cancer sp. } \\
\text { Chionoecetes bairdi Rathbun, } 1924 \\
\text { Liocarcinus holsatus (Fabricius, 1798) } \\
\text { Metacarcinus magister (Dana, 1852) }\end{array}$ & Tellina sp. & Tectonatica sp. & 54 \\
\hline Crab Group I $(n=11)^{\mathrm{A}}$ & Tellinella listeri (Röding, 1798) & Sinum perspectivum $\left(\right.$ Say, 1831) ${ }^{\mathrm{A}}$ & 55 \\
\hline Crab Group I $(\mathrm{n}=11)^{\mathrm{A}}$ & Trachycardium isocardia (L., 1758) & Sinum perspectivum $(\text { Say, } 1831)^{\mathrm{A}}$ & 56 \\
\hline Cancer sp. & Tresus sp. & Tectonatica sp. & 57 \\
\hline $\begin{array}{l}\text { Cancer sp. } \\
\text { Chionoecetes bairdi Rathbun, } 1924 \\
\text { Chionoecetes opilio (O. Fabricius, 1788) } \\
\text { Erimacrus isenbeckii (J. F. Brandt, 1848) } \\
\text { Paralithodes sp. }\end{array}$ & Yoldia sp. & Tectonatica sp. ${ }^{\mathrm{A}}$ & 58 \\
\hline
\end{tabular}

Notes: Crab and gastropod species in the same row (i.e., same bivalve prey 'cluster') followed by the same superscript letters were recorded in the GloBI database as undergoing trophic interactions; for Crab Guilds I-IV, not all crabs in each Guild were recorded to consume the indicated gastropod (see Table 2). Species marked with an '*' were previously reported to undergo K-S interactions (see Table 1).

'node' of a trophic overlap 'cluster' linking its crab and gastropod predators. These 'clusters' included from 1-8 different gastropod species each, and from 1-19 crab species, and thus represented pairs or groups of crab and gastropod species that could potentially undergo K-S and other interactions while feeding on their shared bivalve prey.

Several 'clusters' contained the same overlapping assemblages of the same 9-19 specific crab species, so for the ease of presenting the results these large, recurrent crab assemblages were grouped together under the names 'Crab Groups I-IV' [Table 2; the number of 'clusters' containing Crab Group I ( $\mathrm{n}=11)$, II $(\mathrm{n}=15)$, III $(\mathrm{n}=19)$, and IV $(\mathrm{n}=9)$ was $17,11,1$, and 4 , respectively]. No such 'Groups' were found for the gastropod species obtained.

To further explore and discuss the results obtained, another series of queries was made using the GloBI database on 7 May 2019, but this time for pairs of crab (Anomura and Brachyura) and gastropod (Buccinidae, etc.) species recorded as 'interacts with' one another. The results obtained were then compared to the trophic overlap 'clusters' described above to determine whether any pairs of crabs and gastropods in these 'clusters' are known to eat and/ or undergo other interactions (e.g., competition) with one another. This was done because such congruence would signal the occurrence of IGP and likely BMIIs/TMIIs and indirect interactions between these species, which have been associated with crab-gastropod K-S interactions in past studies (e.g., Morissette \& Himmelman 2000, Herbert et al. 2009, Fodrie et al. 2012, Quinn et al. 2012), and so provides further evidence of potential K-S interactions among these species.

\section{Results}

In total, 58 trophic overlap 'clusters' were identified, each with one bivalve species that was consumed by both one or more species of shell-boring gastropod and one or more crab species as their 'node' (Table 3). These bivalve prey were distributed among 10 bivalve orders in 3 of the 4 bivalve subclasses, specifically Heterodonta (5 orders), Protobranchia (1 order), and Pteriomorpha (4 orders) (Tables 3 and 4). In these 'clusters,' species in the following bivalve orders formed the 'nodes': in the Heterodonta, the orders Adapedonta (razor shells or jackknife clams), Cardiida (cockles and giant clams), Lucinida (cleft clams), Myida (soft-shell clams, geoducks, and shipworms), and Venerida (hard clams); in the Protobranchia, the order Nuculanida; and in the Pteriobranchia, the orders Arcida (ark shells), Limida (file clams), Mytilida (marine mussels), and Ostreida (oysters) (Tables 3 and 4).

Each of the bivalve prey species identified as trophic overlap 'nodes' in this survey was consumed by from 1 to 19 different crab species and from 1 to 8 different gastropod species (Table 3). The gastropods found herein to share the same bivalve prey with crabs included a total of 36 species, comprising 9 species of Buccinidae, 1 of Pisaniidae (previously considered a buccinid whelk), 14 of Muricidae, 7 of Naticidae, and 5 of Cymatiidae that were previously classified as Ranellidae (Tables 3 and 4). The crabs identified herein as sharing bivalve prey with shellboring gastropods (Tables 3 and 4) included a total of 67 different species, including 12 in the infraorder Anomura (3 and 9 in the superfamilies Lithodoidea and Paguroidea, respectively; Table 4) and 55 in the infraorder Brachyura 
Table 4. Number of species (\# spp.) in different gastropod, bivalve, and crab families (and higher taxa) found in this study to potentially undergo tritrophic K-S interactions.

\begin{tabular}{|c|c|c|c|c|c|c|c|}
\hline Order & Superfamily & Family & \# spp. & Infraorder & Superfamily & Family & \# spp. \\
\hline Gastropods & & & & Crabs & & & \\
\hline \multirow[t]{2}{*}{ Littorinimorpha } & Naticoidea & Naticidae & 7 & Anomura & Lithodoidea & Lithodidae & 3 \\
\hline & Tonnoidea & $\begin{array}{l}\text { Cymatiidae } \\
\text { (formerly Ranellidae) }\end{array}$ & 5 & & Paguroidea & Diogenidae & 3 \\
\hline \multirow[t]{3}{*}{ Neogastropoda } & Buccinoidea & Buccinidae & 9 & & & Paguridae & 6 \\
\hline & Muricoidea & $\begin{array}{l}\text { Pisaniidae } \\
\text { (formerly Buccinidae) }\end{array}$ & 1 & Brachyura & Aethroidea & Belliidae & 1 \\
\hline & & Muricidae & 14 & & Calappoidea & Calappidae & 2 \\
\hline Bivalves & & & & & & Carpiliidae & 1 \\
\hline Adapedonta & Solenoidea & Pharidae & 1 & & Cancroidea & Cancridae & 6 \\
\hline \multirow[t]{3}{*}{ Cardiida } & Cardioidea & Cardiidae & 7 & & Cheiragonoidea & Cheiragonidae & 1 \\
\hline & Tellinoidea & Donacidae & 1 & & Grapsoidea & Grapsidae & 2 \\
\hline & & Tellinidae & 4 & & & Varunidae & 3 \\
\hline Lucinida & Lucinoidea & Lucinidae & 5 & & Hymenosomatoidea & Hymenosomatidae & 1 \\
\hline Myida & Myoidea & Myidae & 1 & & Majoidea & Epialtidae & 2 \\
\hline \multirow[t]{4}{*}{ Venerida } & Chamoidea & Chamidae & 2 & & & Majidae & 2 \\
\hline & Mactroidea & Mactridae & 2 & & & Oregoniidae & 4 \\
\hline & Ungulinoidea & Ungulinidae & 2 & & Ocypodoidea & Macrophthalmidae & 2 \\
\hline & Veneroidea & Veneridae & 12 & & & Ocypodidae & 1 \\
\hline \multirow[t]{2}{*}{ Nuculanida } & Nuculanoidea & Nuculanidae & 1 & & Pilumnoidea & Pilumnidae & 10 \\
\hline & & Yoldiidae & 1 & & Pinnotheroidea & Pinnotheridae & 2 \\
\hline \multirow[t]{2}{*}{ Arcida } & Arcoidea & Arcidae & 4 & & Portunoidea & Carcinidae & 3 \\
\hline & & Noetiidae & 1 & & & Ovalipidae & 1 \\
\hline Limida & Limoidea & Limidae & 3 & & & Polybiidae & 3 \\
\hline Mytilda & Mytiloidea & Mytilidae & 6 & & & Portunidae & 3 \\
\hline \multirow[t]{3}{*}{ Ostreida } & Ostreioidea & Ostreidae & 3 & & Xanthoidea & Panopeidae & 3 \\
\hline & Pterioidea & Pteriidae & 2 & & & Pseudorhombilidae & 1 \\
\hline & & & & & & Xanthidae & 1 \\
\hline
\end{tabular}

(belonging to 22 families in 12 superfamilies; Table 4). The majority of bivalves were listed as being consumed by only 1 (31 bivalves) or 2 (19 bivalves) gastropod species, while a handful of others were consumed by 3 gastropods (6 bivalves), and one bivalve each was consumed by 4 or 8 gastropods (Table 3 ). On the other hand, only 6 bivalves each were listed as only being eaten by 1 or 2 crab species, respectively, while a few were consumed by $3,4,5$, or 7 crabs $(2,4,4$, and 1 bivalves, respectively), but the majority (35) of bivalves were preyed upon by 9 or more different crab species (Table 3). Of the 6 and 19 bivalves that were consumed by 9 and 11 crabs, respectively, all of the latter and all but two of the former bivalve species were consumed by Crab Groups I and IV, respectively (Table 3), while all 11 and 1 bivalves consumed by 15 and 19 crabs each were eaten by Crab Groups II and III, respectively (Table 3). Therefore, most crabs were only recorded as sharing bivalve prey with 1 or 2 gastropod species, whereas most gastropods shared their bivalve prey with 9-19 different crab species (Table 3).

Crab Groups I and IV only ever shared bivalve prey with one species of shell-boring gastropod, the naticid Sinum perspectivum (Say, 1831) (Table 3). Crab Group II shared all its bivalve prey with the former ranellids Monoplex pilearis (L., 1758) and Monoplex vespaceus (Lamarck, 1822), and also shared two bivalves with the pisaniid (former buccinid) Gemophos tinctus (Conrad, 1846) (Table 3). Even though Crab Group III contained most of the same species as Crab Groups I, II, and IV (Table 2), it shared its bivalve prey with 3 different shell-boring gastropod species, specifically the muricid Dermomurex pauperculus (C. B. Adams, 1850) and the former ranellids Cymatium femorale (L., 1758) and Monoplex nicobaricus (Röding, 1798) (Table 3). Crab Groups I and II fed on diverse arrays of bivalve prey in the orders Cardiida, Lucinida, Venereida, and Arcida (Group I) and Venerida, Arcida, and Ostreida (Group II) (Table 3), while Crab Group IV was only recorded as consuming species of Lucinida (Table 3). Crab Group III was only recorded as feeding on the mytilid Modiolus americanus (Leach, 1815) (Table 3).

Among the potential interactions identified in this survey, five species clusters (or six species pairs) of the nine 
species groups (or 11 pairs) among which crab-gastropod K-S interactions were previously observed (Table 1) were recovered (Table 3). All other species groups in the trophic overlap 'clusters' represented novel systems in which K-S interactions could occur, but do not appear to have been reported previously.

Among the various pairs and groups of crabs and shellboring gastropods with shared bivalve prey identified herein, many have been reported to undergo trophic interactions in which the crabs consumed the gastropods, therefore representing cases of IGP and introducing the possibility that these gastropods undergo BMIIs or other kinds of TMIIs in response to crab presence. Such species pairs also represent cases in which crab-gastropod K-S interactions are likely to occur. Specifically, 334 (38.3\%) whelk-crab pairs in different prey 'clusters' were identified for which previous trophic interactions had been reported, including 275 pairs in 'clusters' containing Crab Groups I-IV and 59 pairs in other prey 'clusters' (Table 3). Crab Groups I-IV all contained crab species that were reported to consume the 1-3 gastropod species with which these Groups shared bivalve prey, although in no case were all of the crabs in these Groups reported to eat the gastropod(s) in their trophic overlap 'clusters' (Table 2). Specifically, 7 each of the 11 and 9 crabs in Crab Groups I and IV, respectively, were previously reported to undergo trophic interactions with Sinum perspectivum, 11 of the 15 crabs in Crab Group II were reported to feed on Monoplex pilearis and M. vespaceus, and 7 of the 19 crabs in Crab Group III were reported to eat all three gastropods with which it shared bivalve prey (Table 2).

\section{Discussion}

The present survey identified 58 bivalve molluses whose crab and shell-boring gastropod predators may undergo IGP, TMIIs/BMIIs, and thus-far unreported K-S interactions with each other. Including the 'clusters' containing Crab Groups, these represented 871 tritrophic bivalvecrab-gastropod systems, belonging to diverse taxa from around the world within which these interactions may occur. What is more, for the vast majority ( $>99 \%$ ) of these crab-gastropod pairs, no previous reports of K-S interactions between them when feeding on the same bivalve prey were found (Table 1 and references therein). Therefore, a wide array of tritrophic systems was identified within which future studies should look for novel crab-gastropod K-S interactions where the identified bivalves, crabs, and gastropods co-occur to confirm whether such interactions are widespread in nature, and if so to better quantify their consequences, factors mediating their occurrence, and so on.

Particular species were found in this survey that may be especially fruitful subjects for future research on bivalvecrab-gastropod interactions, including K-S interactions, as they were connected to a wide array of other species. For example, the predatory naticid Sinum perspectivum, also known as the white baby ear and found in the Caribbean Sea and along the tropical Atlantic coasts of North and South America (WoRMS Editorial Board 2019), shared 17 and 4 bivalve prey species with Crab Groups I and IV, respectively, which were assemblages of 11 and 9 tropical crabs and hermit crabs. There is thus much potential to investigate K-S interactions between this gastropod and several crab taxa in the Atlantic and Caribbean regions. The former ranellids Monplex pilearis and M. vespaceus (Cymatiidae), also known as the hairy triton and dwarf hairy triton, respectively, are found in the Atlantic, Indian, and Pacific Oceans and known to feed on bivalves (WoRMS Editorial Board 2019), and shared 11 bivalve prey taxa with the 15 tropical crabs and hermit crabs in Crab Group II. These gastropods thus could also be investigated for K-S interactions involving many bivalve and crab species. Several temperate bivalve genera, such as Macoma, Mytilus, Mya, and Yoldia were also consumed by relatively high numbers of anomuran and brachyuran crab taxa, as well as gastropods in the naticid genus Tectonatica. These bivalve prey could support food webs in which K-S interactions are likely, and gastropods in the genus Tectonatica could also be investigated and would likely be found to undergo $\mathrm{K}-\mathrm{S}$ interactions with many different crabs, perhaps when feeding on many different bivalves. Further, due to the wide trophic niches of many crabs, many of the species and genera of crabs identified in this survey fed on multiple bivalves, and thus interacted with several shell-boring gastropods.

It is worth noting that the survey done in the present study likely did not cover all crab-gastropod pairs and bivalve prey among which K-S interactions could occur. This was because this survey used a database that relies on users to upload reports of species interactions (Poelen et al. 2014), and is thus subject to the users' knowledge since, if only users with knowledge of or access to information on certain taxonomic groups or geographic regions have contributed to the database, there will be gaps in the information therein. For example, no records of bivalve consumption or consumption of the same bivalves as any crab taxon were found for several gastropod groups (see Materials and methods), but given the wide diversity of crabs, gastropods, and bivalves in the world and the fact that some members of these groups are known to consume bivalves and/or compete with crabs for other types of shared food (e.g., Powers \& Kittinger 2002, Iyengar et al. 2008, Chiu et al. 2011, Pinotti et al. 2014, Davenport et al. 2015), such gaps more likely reflect the limitations of the database than the actual absence of trophic overlap (and potential K-S interactions) between these gastropods and crabs. Therefore, while the species groups identified herein are systems in which crab-gastropod K-S interactions likely occur, other crab and shell-boring gastropod taxa that feed on the same bivalves beyond those identified in this survey should be investigated for the occurrence of these interactions as well. 
Importantly, if future studies do pursue the question of whether K-S interactions occur among the crab and gastropod 'clusters' identified herein, they must be careful to distinguish between kleptoparasitism and scavenging because these two types of interactions have differing effects on the predators involved: kleptoparasitism is $+/-$ and scavenging is $+/ 0$ for the crab and gastropod, respectively (Quinn \& Boudreau 2016). Almost no previous study reporting crab-gastropod K-S interactions (Table 1) was able to definitively conclude whether kleptoparasitism and/ or scavenging had occurred because they inferred the occurrence of K-S interactions based on the post mortem condition of bivalve prey shells (Ishida 2004, Boudreau et al. 2013, Quinn \& Boudreau 2016). It is likely that both types of interactions occur in nature, but it is conceivable that under some circumstances one occurs more often than the other, for example because the amount and quality of prey biomass obtained by kleptoparasitism is greater or less variable than that obtained by scavenging (Quinn \& Boudreau 2016) or because of relative predator feeding rates (Herbert et al. 2009, Wong et al. 2012). The relative benefits of kleptoparasitism vs. scavenging to the crab, as well as the actual impacts of these on the gastropods and prey, have also not been quantified (but see estimates by Quinn \& Boudreau 2016), although they should be, but doing so will require careful observation to distinguish between these interactions. To make this distinction, the interactions have to be directly observed, either by eye or by video camera (Boudreau et al. 2013). Unfortunately, this means studies need to be more difficult and costly, but given the number of species groups identified herein as potential candidates to undergo K-S interactions, it should be possible for some future studies to address this question as more crab-gastropod K-S interactions are studied.

The present study focused on the potential for crabs to either kleptoparasitize gastropods by stealing bivalve prey from them, or to scavenge on bivalves abandoned by shellboring gastropods (i.e., K-S interactions). However, related interactions may also occur that might also be worth investigating with less restrictive search filters. For example, many other decapod crustacean groups, particularly the clawed lobsters and crayfishes (Astacidea) and clawless lobsters (Achelata), also consume bivalve prey (e.g., Elner \& Campbell 1987, Barclay et al. 2006), and thus may also undergo K-S interactions with gastropods. Clawed lobsters have much larger and stronger chelae than most crabs do, so they may be less likely to benefit by or use K-S interactions than crabs (Juanes 1992, Enderlein et al. 2003), although such interactions may still be important for smaller, younger lobsters, as with crabs (Elner \& Hughes 1978, Smallegange et al. 2008, Quinn et al. 2012, Boudreau et al. 2013). Both crabs and shell-boring gastropods also consume wide ranges of prey, as the same species may prey on barnacles, bivalves, periwinkles, etc. and also scavenge carrion, and thus more generalized forms of kleptoparasitism and scavenging interactions likely do occur among crabs and gastropods, beyond just when they have shared bivalve prey. The Eulimidae are drilling gastropods that parasitize echinoderms, which are subject to crab predation, and thus a form of K-S interaction could also occur when a crab feeds on a eulimid's host (assuming the gastropod is not eaten itself!) (de Souza \& Pimenta 2019, WoRMS Editorial Board 2019); this would be interesting to study. Gastropods in the family Nassariidae are also known to compete with crabs as scavengers for the same carrion (Averbuj et al. 2012, Davenport et al. 2015, 2016), and such interactions could manifest as K-S interactions under some circumstances. Investigating trophic overlap between crabs and shell-boring gastropods when they feed on different types of food and prey may also allow K-S interactions to be detected among herbivorous snails and crabs, such as between terrestrial snails [e.g., 'Pulmonata,' some of which are known borers (Kowalewski 2002)] and land crabs feeding on the same plant materials (e.g., Fratini et al. 2011).

This survey (and most previous studies reporting crabgastropod K-S interactions; Table 1) considered the case in which a crab kleptoparasitizes or scavenges prey from a gastropod, which is usually more likely than the reverse order of events (i.e., a gastropod steals or scavenges prey initially attacked by a crab) because crabs tend to be larger, more aggressive, and more rapidly feeding than snails, can prey on gastropods, and also leave little prey tissue behind after feeding to be scavenged (Smallegange et al. 2010, Quinn et al. 2012, Wong et al. 2012, Boudreau et al. 2013). However, in cases involving relatively large and fast-moving and -feeding gastropods and/or smaller/slower crabs, then the reverse order of events may be probable (Morissette \& Himmelman 2000, Morton \& Yuen 2000, Fodrie et al. 2012, Walsh 2016). For example, buccinid whelks like Buccinum undatum L., 1758 are relatively large, fast, and aggressive, and are known to kleptoparasitize other predators feeding on bivalves (Nielsen 2012), such as sea stars (Morissette \& Himmelman 2000) and possibly crabs (Walsh 2016; Table 1). In such cases, the whelk may be unable to reach the bivalve prey (e.g., a clam) because it buries itself in the sediment, but after its removal by the crab it is then made available to the whelk (Morissette \& Himmelman 2000). In principle, the crab-gastropod pairs and 'clusters' identified herein could undergo K-S interactions in either order depending on the circumstances of the species involved. Further studies to identify these species' $\mathrm{K}-\mathrm{S}$ interactions could provide general rules or theories to help understand and predict the 'direction' of K-S interactions and what factors mediate it (Iyengar 2008, Quinn \& Boudreau 2016), which would provide interesting and useful information for understanding the ecology of these aquatic organisms.

The results of the present study represent potential materials for future reviews (sensu Ates 2017) that could investigate a number of interesting questions. Since K-S interactions have been observed involving both native and 
invasive predator species, but less so (thus far) with invasive species (Table 1), it might be worth investigating whether K-S interactions are more or less likely to occur in invaded communities. The wide diversity of prey and predator taxa (Table 4) identified herein also means that K-S could occur (or not) among organisms with different morphologies, habitats, and life habits, so it would be interesting to assess whether the potential (or actual) occurrence of K-S interactions is related to these. For example, cockles (order Cardiida) have thicker, harder shells than many other bivalves, and thus may be less vulnerable to crab predation, although weakening of them by gastropod drilling may mean that K-S interactions also provide more potential benefits to crabs (e.g., making a new prey item accessible to them). Likewise, bivalve prey that live buried in the substrate could interact differently with their crab and gastropod predators, for example being normally comparatively better protected from shell-boring gastropods than crabs, as note above (Morissette \& Himmelman 2000). Lastly, the range of bivalve prey accessible to crab predators is strongly size-dependent (Enderlein et al. 2003, and see above), and thus the occurrence of K-S interactions between crabs and gastropods likely changes with predator ontogeny. A future study combining such information with the data collected herein could elucidate whether and how such factors impact K-S and other interactions among crabs and shell-boring gastropods feeding on bivalves.

Based on previous studies, K-S interactions between crab and gastropod predators of bivalves provide additional food energy for the kleptoparasite/scavenger, and can lead to energetic losses to the host/primary forager (Fodrie et al. 2012); for example, both these interactions could increase crab predation/feeding rates by $22.8-32.1 \%$, while kleptoparasitism (but not scavenging) could substantially reduce whelk feeding rates by $65.4-67.3 \%$ (Quinn \& Boudreau 2016). They also tend to co-occur with IGP situations and TMIIs/BMIIs, meaning that one predator (usually the crab) can have a disproportionately large negative impact on the other (the gastropod) (Wong et al. 2012, Boudreau et al. 2018, Bolton et al. 2019). As gastropods feeding in the absence of crabs can exert comparable mortality on their prey to that that occurs with gastropods and crabs together (due to their higher numbers, and despite their smaller sizes and slower feeding) (Boudreau \& Hamilton 2012), these interactions are likely important components of the ecological 'balance' within aquatic food webs that prevent the shared prey at the base of the food web from being wiped out by excessive predation (Hamilton 2000, Trussell et al. 2017). The fact that K-S interactions also allow younger, smaller, and weaker crabs to access larger, more energetically rich bivalve prey items than they could on their own is also likely important to crab growth and population recruitment, and may also permit prey switching to avoid intraspecific competition within or between life history stages (Smallegange et al. 2006, 2008, Boudreau et al. 2013). Relative predator size, aggression, feeding rate, prey availability and size, and other factors likely determine whether crab-gastropod K-S interactions can occur, and if so how (e.g., in what 'direction'). However, these interactions have not been reported or studied much previously, so we currently lack the data to develop general theories concerning their occurrence, nature, or impacts. This is an important shortcoming that should be resolved.

Identifying K-S interactions among crabs and gastropods may be important to assessing possible impacts of future changes on or caused by these taxa. Given that bivalves and their invertebrate predators are ectotherms, climate change has the potential to impact their trophic interactions, including K-S ones, for example by altering predator and prey growth and feeding rates (e.g., Miller 2013, Klompmaker et al. 2019). Many crab and gastropod taxa actually are or could become invasive species, and thus their ability to undergo K-S and other interactions may also be important in determining their impacts on native communities (Chiba \& Sato 2013, Quinn \& Boudreau 2016). Given that the IGP, TMIIs/BMIIs, K-S interactions, etc. that result from trophic overlap between predators have important impacts on ecosystem and food web processes (Sih et al. 1985, Trussell et al. 2003, 2017, Boudreau \& Hamilton 2012) and are subject to future changes (Miller 2013, Klompmaker et al. 2019), it is important that these interactions are better understood now, so that if and when they change we can understand why this has happened and perhaps mitigate any negative impacts. The present study generated a global database of crab and gastropod species with shared bivalve prey that could undergo such interactions, which will help guide future studies to investigate this topic.

\section{Acknowledgements}

This study was made possible by the creators, managers, and contributors of the Global Biotic Interactions (GloBI) database, whom I thank for making this information freely available and easily accessible online. I thank Melanie R. Boudreau, Melanie L. Boudreau, Diana Hamilton, and Jeff Clements for their input to past discussions on shell-boring gastropods and K-S interactions, Marcelo M. Rivadeneira, Deepak Samuel Vijay Kumar, and Syed Abbas Jafar for sharing useful information and papers, anonymous reviewers for helpful feedback on earlier versions of this paper, and Monica Liu for support during the paper's initial preparation. The University of New Brunswick (Saint John Campus) provided access to literature and resources that aided in this study.

\section{References}

Arim M, Marquet, PA (2004) Intraguild predation: a widespread interaction related to species biology. Ecol Lett 7: 557-564.

Aschaffenberg MD (2008) Different crab species influence feed- 
ing of the snail Nucella lapillus through trait-mediated indirect interactions. Mar Ecol 29: 348-353.

Ates RML (2017) Benthic scavengers and predators of jellyfish, material for a review. Plankton Benthos Res 12: 71-77.

Averbuj A, Palomo G, Brogger MI, Penchaszadeh PE (2012) Diet and feeding of the nassariid Buccinanops cochlidium from northern Patagonia, Argentina. Aquat Biol 17: 261-268.

Barclay MC, Irvin SJ, Williams KC, Smith DM (2006) Comparison of diets for the tropical spiny lobster Panulirus ornatus: astaxanthin-supplemented feeds and mussel flesh. Aquac Nutr 12: 117-125.

Barnard CJ, Sibly RM (1981) Producers and scroungers: a general model and its application to feeding flocks of house sparrows. Anim Behav 29: 543-550.

Bolton DK, Clark GF, Johnston EL (2019) Novel in situ predator exclusion method reveals the relative effects of macro and mesopredators on sessile invertebrates in the field. J Exp Mar Biol Ecol 513: 13-20.

Boudreau ML, Boudreau MR, Hamilton DJ (2013) The influence of body size on foraging facilitation and kleptoparasitic behavior in the green crab (Carcinus maenas). J Exp Mar Biol Ecol 449: 330-334.

Boudreau ML, Scrosati RA, Wong MC (2018) Predator (Carcinus maenas) nonconsumptive limitation of prey (Nucella lapillus) feeding depends on prey density and predator cue type. J Ethol 36: 259-264.

Boudreau MR, Hamilton DJ (2012) Seasonal variation in effects of multiple predators on an intertidal mussel bed: implications for interpretation of manipulative experiments. Mar Ecol Prog Ser 465: 137-153.

Carriker MR, Williams LG (1978) The chemical mechanism of shell dissolution by predatory boring gastropods: a review and an hypothesis. Malacologia 17: 143-156.

Chiba T, Sato, S (2013) Invasion of Laguncula pulchella (Gastropoda: Naticidae) and predator-prey interactions with bivalves on the Tona coast, Miyagi prefecture, northern Japan. Biol Invasions 15: 587-598.

Chiu JMY, Shin PKS, Yang FY, Cheung SG (2011) Can a scavenging gastropod with a mussel conspecific diet induce antipredator defence in the mussel Perna viridis? J Exp Mar Biol Ecol 401: 85-88.

Cockrell ML, Bernhardt JR, Leslie HM (2015) Recruitment, abundance, and predation on the blue mussel (Mytilus edulis) on northeastern estuarine rocky shores. Ecosphere 6: 18. DOI: 10.1890/ES14-00176.1.

Daleo P, Escapa M, Isacch JP, Ribeiro P, Iribarne O (2005) Trophic facilitation by the oystercatcher Haematopus palliatus Temminick on the scavenger snail Buccinanops globulosum Kiener in a Patagonian bay. J Exp Mar Biol Ecol 325: 27-34.

Davenport J, McCullough S, Thomas RW, Harman L, McAllen R (2015) Nassariid whelks hitch-hiking on Cancer pagurus: phoresis, commensalism or fouling? Mar Biodiv Rec 8: e146. DOI: 10.1017/S1755267215001232.

Davenport J, McCullough S, Thomas RW, Harman L, McAllen R (2016) Behavioural responses of shallow-water benthic marine scavengers to fish carrion: a preliminary study. Mar Freshwat Behav Physiol 49: 301-315.

Dill LM, Heithaus MR, Walters CJ (2003) Behaviorally mediated indirect interactions in marine communities and their conservation implications. Ecology 84: 1151-1157.

Elner RW, Campbell A (1987) Natural diets of American lobster Homarus americanus from barren ground and microalgal habitats off southwestern Nova Scotia, Canada. Mar Ecol Prog Ser 37: 131-140.

Elner RW, Hughes RN (1978) Energy maximization in the diet of the shore crab Carcinus maenas. J Anim Ecol 47: 103-116.

Enderlein PS, Wahl M (2004) Dominance of blue mussels versus consumer-mediated enhancement of benthic diversity. J Sea Res 51: 145-155.

Enderlein PS, Moorthi S, Röhrscheidt H, Wahl M (2003) Optimal foraging versus shared doom effects: interactive influence of mussel size and epibiosis on predator preference. J Exp Mar Biol Ecol 292: 231-242.

Fodrie FJ, Brodeur MC, Toscano BJ, Powers SP (2012) Friend or foe: conflicting demands and conditional risk taking by opportunistic scavengers. J Exp Mar Biol Ecol 422-423: 114-121.

Fratini S, Sacchi A, Vannini M (2011) Competition for mangrove leaf litter between two East African crabs, Neosarmatium meinerti (Sesarmidae) and Cardisoma carnifex (Gecarcinidae): a case of kleptoparasitism? J Ethol 29: 481-485.

Hamilton DJ (2000) Direct and indirect effects of predation by common eiders and abiotic disturbance in an intertidal community. Ecol Monogr 70: 21-43.

Herbert GS, Dietl GP, Fortunato H, Simone LRL, Sliko J (2009) Extremely slow feeding in a tropical drilling ectoparasite, $\mathrm{Vi}$ tularia salebrosa (King and Broderip, 1832) (Gastropoda: Muricidae), on molluscan hosts from Pacific Panama. Nautilus 123: 121-136.

Ishida S (2004) Initial predation and parasitism by muricid whelks demonstrated by the correspondence between drilled holes and their apparent enveloper. J Exp Mar Biol Ecol 305: 233-245.

Iyengar EV (2008) Kleptoparasitic interactions throughout the animal kingdom and a re-evaluation, based on participant mobility, of the conditions promoting the evolution of kleptoparasitism. Biol J Linn Soc 93: 745-762.

Iyengar EV, Sitvarin MI, Cataldo M (2008) Function of the flexible periostracal hairs in Trichotropis cancellata (Mollusc, Gastropoda). Invert Biol 127: 299-313.

Juanes F (1992) Why do decapod crustaceans prefer small-sized molluscan prey? Mar Ecol Prog Ser 87: 239-249.

Klompmaker AA, Kelley PH, Chattopadhyay D, Clements JC, Huntley JW, Kowalewski M (2019) Predation in the marine fossil record: studies, data, recognition, environmental factors, and behavior. Earth-Sci Rev 194: 472-520.

Kowalewski M (2002) The fossil record of predation: an overview of analytical methods. Paleontol Soc Papers 8: 3-42.

Liu D, Wang F, Yang C, Hu N, Sun Y (2017) Starvation and a conspecific competitor influence multiple predator effects in a swimming crab (Portunus trituberculatus) - Manila clam (Ruditapes philippinarum) foraging system. J Exp Mar Biol Ecol 495: 35-42.

Liu D, Su X, Wang F, Zhong D, Sun Y, Zhang D (2019) Starvation intensifies the impacts of interspecific interactions on foraging behavior of swimming crab (Portunus trituberculatus). Aquaculture 504: 22-29. 
Miller LP (2013) The effect of water temperature on drilling and ingestion rates of the dogwhelk Nucella lapillus feeding on Mytilus edulis mussels in the laboratory. Mar Biol 160: 1489-1496.

Morgan SG, Gravem SA, Lipus AC, Grabiel M, Miner BG (2016) Trait-mediated indirect interactions among residents of rocky shore tidepools. Mar Ecol Prog Ser 552: 31-46.

Morissette S, Himmelman JH (2000) Subtidal food thieves: interactions of four invertebrate kleptoparasites with the sea star Leptasterias polaris. Anim Behav 60: 531-543.

Morton B, Yuen WY (2000) The feeding behaviour and competition for carrion between two sympatric scavengers on a sandy shore in Hong Kong: the gastropod, Nassarius festivus (Powys) and the hermit crab, Diogenes edwardsii (De Haan). J Exp Mar Biol Ecol 246: 1-29.

Morton B (2011) Predator-prey-scavenging interactions between Nucella lapillus, Carcinus maenas and Eulalia viridis all exploiting Mytilus galloprovincialis on a rocky shore recovering from tributyl-tin (TBT) pollution. J Nat Hist 45: 2397-2417.

Nielsen C (2012) Observation on Buccinum undatum L. attacking bivalves and on prey responses, with a short review on attack methods of other prosobranchs. Ophelia 13: 87-108.

Pinotti RM, Minasi DM, Colling LA, Bemvenuti CE (2014) A review on microbenthic relationships along subtropical sandy shores in southernmost Brazil. Biota Neotrop 14: e20140069. DOI: 10.1590/1676-06032014006914.

Poelen JH, Simons JD, Mungall CJ (2014) Global biotic interactions: an open infrastructure to share and analyze speciesinteraction datasets. Ecol Inform 24: 148-159.

Polis GA, Holt RD (1992) Intraguild predation: the dynamics of complex trophic interactions. Trends Ecol Evol 7: 151-154.

Powers SP, Kittinger JN (2002) Hydrodynamic mediation of predator-prey interactions: differential patterns of prey susceptibility and predator success explained by variation in water flow. J Exp Mar Biol Ecol 273: 171-187.

Quinn BK, Boudreau MR (2016) Kleptoparasitism and scavenging by the invasive green crab (Carcinus maenas) have different impacts on native species. Mar Biol 163: 186. DOI: 10.1007/s00227-016-2964-1.

Quinn BK, Boudreau MR, Hamilton DJ (2012) Inter- and intraspecific interactions among green crabs (Carcinus maenas) and whelks (Nucella lapillus) foraging on blue mussels (Mytilus edulis). J Exp Mar Biol Ecol 412: 117-125.
Seed R (1976) Ecology. In: Marine Mussels: Their Ecology and Physiology (ed Bayne BL). Cambridge University Press, Cambridge, UK, pp. 13-26.

Sih A, Crowley P, McPeek M, Petranka J, Strohmeier K (1985) Predation, competition, and prey communities: a review of field experiments. Ann Rev Ecol Syst 16: 269-311.

Smallegange IM, Hidding B, Eppenga JMA, van der Meer J (2008) Optimal foraging and risk of claw damage: how flexible are shore crabs in their prey size selectivity? J Exp Mar Biol Ecol 367: 157-163.

Smallegange IM, van der Meer JMA, Sabelis MW (2010) “Takeaway" foraging spatially uncouples predator and prey-attack distributions. J Anim Ecol 79: 769-776.

Smallegange IM, van der Meer J, Kurvers RHJM (2006) Disentangling interference competition from exploitation competition in a crab-bivalve system using a novel experimental approach. Oikos 113: 157-167.

de Souza LS, Pimenta AD (2019) Eulimacrostoma gen. nov., a new genus of Eulimidae (Gastropoda, Caenogastropoda) with description of a new species and reevaluation of other western Atlantic species. Zoosystem Evol 95: 405-415.

Trussell GC, Matassa CM, Ewanchuk PJ (2017) Moving beyond linear food chains: trait-mediated indirect interactions in a rocky intertidal food web. Proc R Soc B 284: 20162590. DOI:10.1098/rspb.2016.2590.

Trussell GC, Ewanchuck PJ, Bertness MD (2003) Trait-mediated effects in rocky intertidal food chains: predator risk cues alter prey feeding rates. Ecology 84: 629-640.

Vadas RL, Burrows MT, Hughes RN (1994) Foraging strategies of dogwhelks, Nucella lapillus (L.): interacting effects of age, diet and chemical cues to the threat of predation. Oecologia 100: 439-450.

Walsh S (2016) Kleptoparasitic behaviour of Buccinum undatum in the presence of Carcinus maenas. In: BIOL 3933 (Directed Studies in Marine Sciences) 2019 Final Reports, University of New Brunswick, Saint John, NB, Canada.

Wong MC, d'Entremont J, Barbeau M (2012) An approach for quantifying effects of multiple predators that forage on different time scales. J Exp Mar Biol Ecol 420-421: 100-109.

WoRMS Editorial Board (2019) World Register of Marine Species. Available from: http://www.marinespecies.org at VLIZ. Accessed 2019-06-04. DOI: 10.14284/170. 